\title{
DIVIDING A SYSTEM INTO ALMOST UNIDTRECTIONAL BLOCKS
}

\section{Wataru Mayeda}

Coordinated Science Laboratory

University of Illinois

Urbana, IL 61801 U.S.A. $\left(\begin{array}{c}\text { Dept. of Integrated Arts and Sciences } \\ \text { Hiroshima University } \\ \text { Hiroshima } 730 \text {, Japan }\end{array}\right)$

Abstract. When a system is divided into blocks $B_{1} B_{2} \ldots B_{k} \ldots B_{m}$ such that the existence of failure in $B_{k}$ will not interfare required performance of blocks $B_{1} B_{2} \ldots$ and $B_{k-1}$, then these blocks are called unidirectional blocks. To determine failure elements in a system, unidirectional blocks $B_{1} B_{2} \ldots$ will be tested one by one in the order to find a block containing failure first, then diagnose the block to locate failure elements. It is easily seen that this process is simpler than testing a system as a whole to determine the location of failure elements by one procedure. Unfortunatley, most of systems can not be divided into unidirectional blocks. However, it is possible to divide a system into almost unidirectional blocks $B_{1} B_{2} \ldots B_{k} \ldots B_{m}$ such that the existence of failure in $B_{k}$ will interfare only slightly to the performance of preceding blocks $B_{1} B_{2} \ldots B_{k-1}$.

\section{Introduction}

As huge quantities of printed circuits are utilized in wide area, diagnosis of electrical networks becomes an extremely important task. Especially, for those high priced printed circuits, it is mone economical to fix those printed circuits when failure occured than renew them. This situation leads to start research on automatic system diagnosis. Since it is not advisable to remove elements in a printed circuit for diagnosis, usually information for diagnosis are only those obtained by measurement of voltages at available nodes. However, sources for such measurements can be either voltages or currents.

\section{Value of Terminal Transmission}

Printed circuits for analog networks usually consists of several blocks of different performances such as local oscillators, mixers and amplifiers. Also each of these blocks may consist of several smaller blocks. For example, an amplifier usually 
consists of several simple amplifier circuits in series. Hence when a failure occurs, it is reasonable to test each block for the existence of a failure, first. Once, a faulty block is found then, the second step is to determine location of failed elements in the block. To accomplish this procedure, a system must be divided into suitable blocks.

Let $B_{1} B_{2} \ldots B_{k} \ldots B_{m}$ be blocks which form a system. Then, these blocks are called unidirectional blocks if a failure of block $B_{k}$ will not interfare a required performance of blocks $B_{1} B_{2} \ldots B_{k-1}$ for all $k=2,3, \ldots$, m. They are called almost unidirectional blocks, if a failure of block $B_{k}$ will have a1most no effect on a required performance of $B_{1} B_{2} \ldots B_{k-1}$ for all $k=2,3, \ldots, m$. Consider an electrical network $N$ containing nodes $0,1,2, \ldots$, $n$ with 0 being the reference node. Let $v_{j}$ be a voltage at node $j$ when a source voltage $v_{i}$ is connected from node $i$ to the reference node 0 . Then a value of transmission from $i$ to $j$ symbolized by $\alpha_{i j}$ is defined as

$$
\alpha_{i j}=F\left(v_{i}, v_{j}\right)
$$

For example, $\alpha_{i j}=v_{j} / v_{i}, \alpha_{i j}=\left|v_{j}\right| /\left|v_{i}\right|$, or $\alpha_{i j}=v_{j}^{2} / v_{i}^{2}$. By taking $i$ and $j$ equal to 1 , $2, \ldots, n$. with $i \neq j$, we have $n ! /(n-2) ! \alpha$ s. Using $\alpha_{i j}$ as a weight of edge from vertex. $j$, we have an oriented graph $G$ corresponding to a given electrical network $N$. As an example, a graph G in Fig. I (a) is obtained from an electrical network $N$ in (b) with $\alpha_{i j}=v_{j} / v_{i}$.

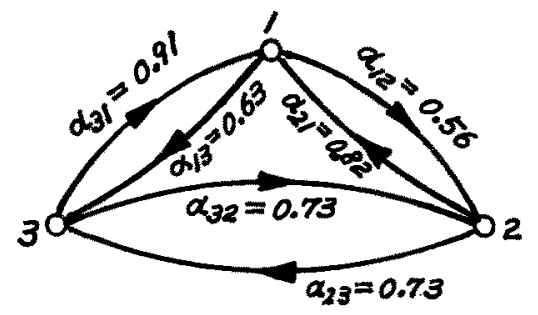

(a) Graph G

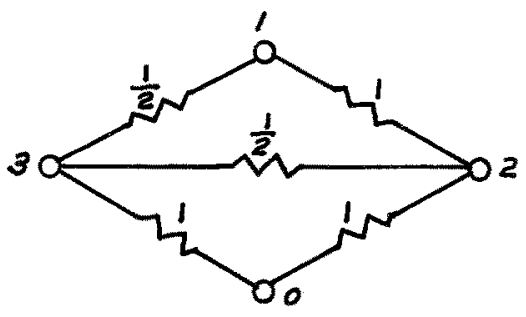

(b) Network N

Fig. I A network $\mathrm{N}$ and corresponding graph $\mathrm{G}$.

Let $g_{p}$ be a subgraph of $G$. Also let vertex $i$ be not in $g_{p}$. Then a value of $\alpha_{i g_{p}}$ from vertex $i$ to subgraph $g_{p}$ is defined as

$$
\alpha_{i g_{p}}=\operatorname{Max}\left\{\alpha_{i p} ; \operatorname{pgg} g_{p}\right\}
$$

Note that $\alpha_{i g_{p}}$ is the largest value of transmission from $i$ to $p$ for all vertices in 
$g_{p}$. Also note that when $\alpha$ 's are not real numbers, we must define a way of ordering $a^{\prime} s$. When we consider an amount of signal being transfered form a node $i$ to a block of a network $\mathrm{N}$, each node in the block will receive from a source at node $i$, we take the largest amount of signal reached at a node among all nodes in the block.

Let $g_{i}$ and $g_{p}$ be subgraphs of $G$ which have not vertices in common. Then a value $\alpha_{g_{i} g_{p}}$ of transmission from $g_{i}$ to $g_{p}$ is defined as

$$
a_{g_{i} g_{p}}=\operatorname{Max}\left\{\alpha_{i p} ; i \epsilon g_{i}, p \in g_{p}\right\}
$$

This is equivalent to taking a value of transmission of signal between blocks as the largest amont of transmission between nodes belong to these blocks.

Finally, we define a value of terminal transmission $\tau_{i j}$ from vertex $i$ to vertex $j$ is defined as

$$
\tau_{i j}=\operatorname{Min}\left\{\alpha_{g_{i} g_{j}} ; a 11 g_{i} \text { s.t. } i \epsilon g_{i}, j \notin g_{i}, g_{j}=\bar{g}_{i}\right\}
$$

where $\bar{g}_{j}$ is the complement of $g_{j}$ obtained by deleting all vertices and edges connected to these vertices in $g_{j}$.

Note that, in general, values $\tau_{i j}$ and $\tau_{j i}$ of terminal transmission will be different. Hence, for a graph $G$ of $n$ vertices, there are $n ! /(n-2) !$ terminal transmission $\tau^{\prime} \mathrm{s}$.

Let $\{\mathrm{T}\}$ be a collection of all terminal transmissions of $G$. Suppose the values of all terminal transmissions are positive real numbers. Then from the smallest one, we can order them as

$$
\tau_{1} \leqslant \tau_{2} \leqslant \ldots \leqslant \tau_{u}
$$

where $u=n ! /(n-2) !$. By the definition of $\tau_{i j}$, one of $\alpha$ 's in $\left\{\alpha_{g_{j}} g_{j} ; a 11 g_{i}\right.$ s.t. $i \in g_{i}$, $\left.j \notin g_{i}, g_{j}=\bar{g}_{i}\right\}$ has the value equal to $\tau_{i j}$. Let $\alpha_{g_{i}} g_{j}$, be the one which is equal to $\tau_{i j}$. Let $S$ be a cutset such that by deleting all edges in $S$ from $G$, we have subgraphs $g_{i}$ and $g_{j}^{\prime}$. This cutset $S$ is called a corresponding cutset of $\tau_{i j}$. For convenience we use the symbol $S\left(\tau_{i j}\right)$ to indicate such a corresponding cutset of $\tau_{i j}$. If there are several $\alpha_{g_{i}^{\prime} g_{j}^{\prime}}$ in $\left\{\alpha_{g_{i} g_{j}}\right.$; all $g_{i}$ s.t. $\left.i \epsilon g_{i}, j g^{\prime} g_{j}, g_{j}=\bar{g}_{i}\right\}$ which are equal to $\tau_{i j}$, there are several corresponding cutsets of $\tau_{i j}$.

For convenience, we say that two cutsets $s_{p}$ and $S_{q}$ do not intersect each other if deletion of all edges in $s_{p}$ and $s_{q}$ from graph $G$ produces exactly three connected subgraphs. Consider two values $\tau_{i}$ and $\tau_{j}$ where $\tau_{i} \leqslant \tau_{j}$. There are corresponding cutsets $S\left(\tau_{i}\right)$ and $S\left(\tau_{j}\right)$ having an important property shown by the following theorem:

Theorem 1: If cutsets $S\left(\tau_{i}\right)$ and $S\left(\tau_{j}\right)$ do intersect each other, there exists another corresponding cutset $S^{\prime}\left(\tau_{j}\right)$ of $\tau_{j}$ which does not interesct with $S\left(\tau_{i}\right)$. 
Proof: Let the values of transmission corresponding to $\tau_{\gamma}$ and $\tau_{j}$ be $\alpha_{p q}$ and $\alpha_{r s}$ respectively. Let $g_{a}, g_{b}, g_{c}$ and $g_{d}$ be four connected subgraphs obtained by deleting all edges in $S\left(\tau_{i}\right)$ and $S\left(\tau_{j}\right)$.

Case 1: Suppose $\alpha_{p q}$ and $\alpha_{r s}$ are connected from $g_{a}$ as shown in Fig. 2.

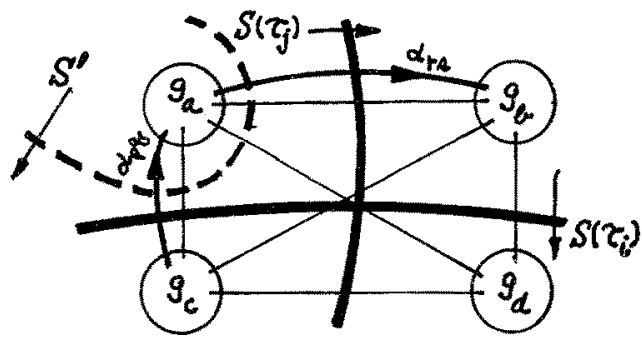

Fig. 2. $S\left(\tau_{i}\right)$ and $S\left(\tau_{j}\right)$.

It is clear from the definition of $\tau_{i}$ that $\alpha_{\mathrm{pq}}$ is the largest among the weights of all edges directed from $g_{a}$ and $g_{b}$ to $g_{c}$ and $g_{d}$ in $S\left(\tau_{i}\right)$. Also $\alpha_{r s}$ is the largest among the weights of all edges in $S\left(\tau_{j}\right)$ directed from $g_{a}$ and $g_{c}$ to $g_{b}$ and $g_{d}$. Since $\tau_{1} \leqslant \tau_{j}, \alpha_{p q} \leqslant \alpha_{r s}$, consider a cutset $S^{\prime}$ in Fig. 2 which contains both $\alpha_{p q}$ and $\alpha_{r s}$. The largest weight of edges in $\mathrm{S}^{\prime}$ directed from $\mathrm{g}_{\mathrm{a}}$ to $\mathrm{g}_{\mathrm{b}}, \mathrm{g}_{\mathrm{c}}$ and $\mathrm{g}_{\mathrm{d}}$ is clearly ${ }^{\mathrm{a}} \mathrm{rs}$ which is equal to $\tau_{j}$. This means that there exists a value of transmission $\alpha_{g_{a}} \bar{g}_{a}$ in $\left\{\alpha_{g_{a} g_{b}}\right.$; all $g_{a}$ s.t. $\left.i \in g_{a}, j / g_{a}, g_{b}=\bar{g}_{a}\right\}$ which is equal to $\tau_{j}$. Note that $\bar{g}_{a}$ consists of $g_{b}, g_{c}, g_{d}$ and all edges connected between them. Hence $S^{\prime}$ is a corresponding cutset of $\tau_{j}$ and $S$ ' does not intersect with $S\left(\tau_{i}\right)$.

The same results can be obtained when the location of $\alpha_{\mathrm{pq}}$ and $\alpha_{\mathrm{rs}}$ are different from those of Case 1 .

When $\tau_{i}=\tau_{j}$, it is possible to have a situation that corresponding cutsets $S\left(\tau_{i}\right)$ and $S\left(\tau_{j}\right)$ are identical. In such a case, we like to state that the corresponding cut sets of $T_{i}$ and $T_{j}$ do not intersect each other. Hence, we define that identical cutsets do not intersect each other. By extending the previous theorem, we have

Theorem 2: Suppose any two of $S\left(\tau_{1}\right), S\left(\tau_{2}\right), \ldots, S\left(\tau_{r}\right)$ do not intersect each other. Also suppose $S\left(\tau_{x+1}\right)$ intersects with some of $S\left(\tau_{1}\right), S\left(\tau_{2}\right), \ldots$ and $S\left(\tau_{x}\right)$. Then there exists another corresponding cutset $S^{\prime}\left(\tau_{r+1}\right)$ of $\tau_{r+1}$ which does not intersect with any of $S\left(\tau_{1}\right), S\left(\tau_{2}\right), \ldots$ and $S\left(\tau_{r}\right)$.

There are relationship among $\tau$ 's given by the next thoerem.

Theorem 3: Let $g_{i}$ and $g_{j}$ be connected subgraph of $G$ obtained by deleting all edges in $S\left(\tau_{i j}\right)$ and $i \epsilon g_{i}$. Then for any pair of vertices $p \epsilon g_{i}$ and $q \epsilon g_{j}$,

$$
\tau_{p q} \leqslant \tau_{i j}
$$


Proof: From the definition of $\tau_{i j}$, that is,

$$
{ }^{T}{ }_{i j}=\operatorname{Min}\left\{\alpha_{i j} ; \text { all } g_{i} \text { s.t. } i \leftarrow g_{i}, j \in g_{i}, g_{j}=\vec{g}_{i}\right\}
$$

There exists a value $\alpha_{i j}$ in $\left\{\alpha_{i j} ;\right.$ all $\left.g_{i} s . t . i \epsilon g_{i}, g_{j}=\bar{g}_{i}\right\}$ such that $\alpha_{i j}$ is equal to $\tau_{i j}$. Consider the definition of $\tau_{p q}$ where

$$
\tau_{p q}=\operatorname{Min}\left\{\alpha_{p q} ; \text { all } g_{p} \text { s.t. } p \in g_{p}, g^{\prime} g_{p}, g_{q}=\bar{g}_{p}\right\}
$$

Since $\mathrm{p}^{\epsilon} \mathrm{g}_{i}$ and $q \epsilon \mathrm{g}_{\mathrm{j}}, \alpha_{i j}$ is in the collection $\left\{\alpha_{\mathrm{pq}} ;\right.$ all $\mathrm{g}_{\mathrm{p}}$ s.t. $\left.\mathrm{p} \epsilon \mathrm{g}_{\mathrm{p}}, q d \mathrm{~g}_{\mathrm{p}}, \mathrm{g}_{\mathrm{q}}=\bar{g}_{\mathrm{p}}\right\}$. Because $\tau_{\mathrm{pq}}$ is the minimum among values in the collection, $\tau_{\mathrm{pq}}$ can not be larger than $\alpha_{i j}\left(=\tau_{i j}\right)$.

Note that when ${ }{ }_{i j}$ is the smallest among all values of terminal transmission,

$$
\tau_{p q}=\tau_{i j}
$$

for all $p \in g_{i}$ and $q \in g_{j}$ *

\section{Semicut and Terminal Transmission}

A corresponding graph $\mathrm{G}$ of a network $\mathrm{N}$ is an oriented graph. So we orient a cutset $S\left(\tau_{i j}\right)$ from $g_{i}$ to $g_{j}$ which are two connected subgraphs obtained by deleting all edges in $S\left(\tau_{i j}\right)$ and $i \in g_{i}$. We form two disjoint subsets of $s\left(\tau_{i j}\right)$ called semicuts as follows:

$$
\begin{aligned}
& s_{i j}\left(\tau_{i j}\right): \text { collection of all edges connected from } g_{i} \text { to } g_{j} \text { in } s\left(\tau_{i j}\right) \\
& s_{j i}\left(\tau_{i j}\right): \text { collection of all edges connected from } g_{j} \text { to } g_{i} \text { in } s\left(\tau_{i j}\right)
\end{aligned}
$$

It is clear that

and

$$
s_{i, j}\left(\tau_{i j}\right) \cup s_{j i}\left(\tau_{i j}\right)=s\left(\tau_{i j}\right)
$$

$$
s_{i j}\left(\tau_{i j}\right) \cap s_{j i}\left(\tau_{i j}\right)=\phi \text {. }
$$

By defining $V\left[s_{i j}\left(\tau_{i j}\right)\right]$ as

$V\left[s_{i j}\left(\tau_{i j}\right)\right]=$ the largest value among the weight of all edges in $s_{i j}\left(\tau_{i j}\right)$, we can see that $\tau_{i j}$ is equal to $V\left[s_{i j}\left(\tau_{i j}\right)\right]$.

Hence,

Theorem 4: Value $\tau_{\mathrm{pg}}$ of terminal transmission is equal to

$$
\tau_{p q}=\operatorname{Min}\left\{V\left(s_{p q}\right) ; s_{p q} \in\left\{S_{p q}\right\}\right\}
$$

where $\left\{\mathrm{S}_{\mathrm{pq}}\right\}$ is a collection of all cutsets separating vertices $\mathrm{p}$ and $\mathrm{q}$. Note that a cutset separating $p$ and $q$ means that two connected subgraphs $g_{p}$ and $g_{q}$ obtained by deleting all edges in the cutset satisfy that $\epsilon \mathrm{g}_{\mathrm{p}}$ and $\mathrm{q} \epsilon \mathrm{g}_{\mathrm{q}}$.

The proof can be obtained direct1y form the definition of $\tau_{\mathrm{pq}}$. By this theorem, if we calculate $V\left[s_{i j}(\tau i j)\right]$ for all cutsets in $G$, we can obtain all values of terminal transmission. As an example, $V\left[s_{i j}\left(\tau_{i j}\right)\right]$ of graph $G$ in Fig. 1 (a) is shown in Fig. 3 from which we can obtain every $\tau_{\mathrm{pq}}$ as 


$$
\begin{aligned}
& \tau_{12}=\operatorname{Min}\left\{V\left(s_{1,23}\right), V\left(s_{13,2}\right)\right\}=\operatorname{Min}\{0.63,0.73\}=0.63 \\
& { }_{13}=\operatorname{Min}\left\{V\left(s_{1,23}\right), V\left(s_{12,3}\right)\right\}=\operatorname{Min}\{0.63,0.73\}=0.63 \\
& \tau_{23}=\operatorname{Min}\left\{V\left(s_{2,13}\right), V\left(s_{12,3}\right)\right\}=\operatorname{Min}\{0.82,0.73\}=0.73 \\
& \tau_{21}=\operatorname{Min}\left\{V\left(s_{2,13}\right), V\left(s_{23,1}\right)\right\}=\operatorname{Min}\{0.82,0.91\}=0.82 \\
& \tau_{31}=\operatorname{Min}\left\{V\left(s_{3,12}\right), V\left(s_{23,1}\right)\right\}=\operatorname{Min}\{0.91,0.91\}=0.91 \\
& \tau_{32}=\operatorname{Min}\left\{V\left(s_{3,12}\right), V\left(s_{13,2}\right)\right\}=\operatorname{Min}\{0.91,0.73\}=0.73
\end{aligned}
$$

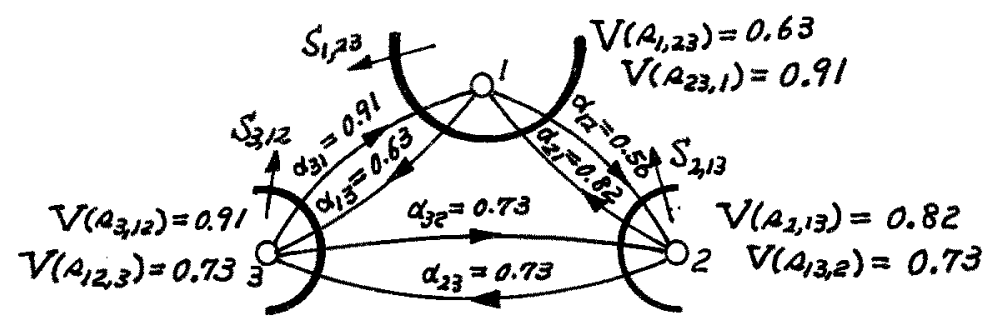

Fig. $\left.3 \quad \mathrm{~V}_{i j}\left(\tau_{i j}\right)\right]$

One way to show all values of terminal transmission is to use a matrix called a terminal transmission matrix symbolized by $T=\left[\tau_{i j}\right]$ where

$$
\tau_{i j}=\left\{\begin{array}{l}
d \quad \text { for } i=j \\
\text { value of terminal transmission from } i \text { to } j \text { for } i \neq j
\end{array}\right.
$$

As an example, of a graph in Fig. 1 (a) is

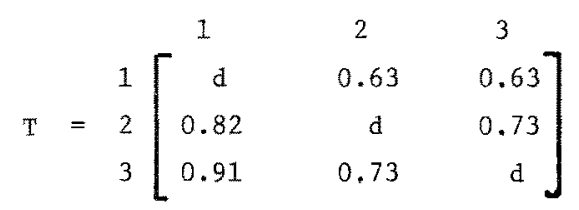

The properties given by those theorems indicate that a terminal transmission matrix has an identical property as a terminal capacity matrix of a communication net ${ }^{4}$. That is, $T$ can be continuously principal partitionable, As an example, T given previously can be continuously partitionable as

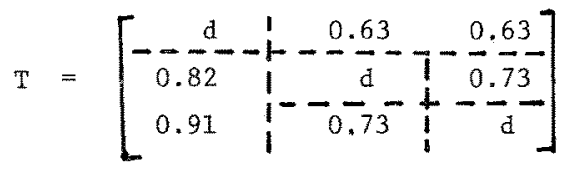


Determination of Almost Unidirectional Blocks

In order to examine the existance of failure by testing blooks one by one starting from block $B_{1}$, then $B_{2}$ and so on, undesired signal from a block $B_{k}$ caused by presence of failure should not influence measurement of block $B_{j}$ for $j<k$. In other woxds, value $\tau_{k j}$ of terminal transmission from a vertex in $B_{k}$ to a vertex in $B_{j}$ should be 0 (or sufficiently sma11). Hence we need to divide graph $G$ into unidirectional blocks (or almost unidirectional blocks). When unidirectional blocks exist, we can see easily that some of $\tau_{i j}$ are 0 . Hence

Theorem 5: Unidirectiona1 blocks exist if and only if there exists semicut $s$ whose value $V[s]$ is 0 .

Let semicuts $s_{1}, s_{2}, \ldots, s_{k}$ be those whose value $v\left(s_{r}\right)=0$. Let cutsets $s_{r}$ correspond to semicut $s_{r}$ for $r=1,2, \ldots, k$. Then by deleting all edges in these cutsets graph $G$ will be divided into connected subgraphs. These subgraphs indicate blocks which form a unidirectional blocks.

We can state Theorem 5 differently by the use of a terminal transmission matrix. When a matrix is principally partitioned as

$$
\left[\begin{array}{c:c}
A & C \\
\hdashline D & B
\end{array}\right]
$$

then $\mathrm{C}$ is called the resultant upper off diagonal matrix. $\mathrm{D}$ is the resultant lower off diagonal matrix and $A$ and $B$ are resultant main diagonal matrices. The name "principal partition" comes because diagonal elements of A and B are diagonal elements of an original matrix. Furthermore all entries of the resultant upper off diagonal matrix consists of identical entry whose value is the smallest among all entries except diagonal elements of given matrix. Continuously principal partition of a matrix means to principal partitioning of a matrix and principal partitioning of all resultant main diagonal matrices produced by previous principal partition. Hence, Theorem 5 can be rewritten as

Theorem 6: If and only if continuously principal partition to a terminal transmission matrix, there are upper off diagonal matrices whose entries are 0 , then there exist unidirectional blocks.

As an example, suppose a terminal transmission matrix is principal partitioned as

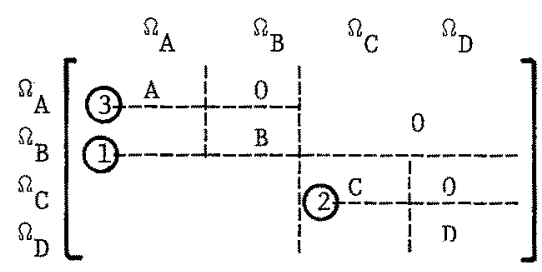


The first principal partition indicated by (1) gives cutset $S_{1}$. The second principal partition indicated by (2) gives cutset $s_{2}$ and the third gives $s_{3}$. Let $g_{A}$ be a subgraph containing vertices in $\Omega_{\mathrm{A}}, g_{\mathrm{B}}$ be a subgraph containing vertices in $\Omega_{\mathrm{B}}$, $\mathrm{g}_{\mathrm{C}}$ containing vertices in $\Omega_{C}$ and $g_{D}$ containing vertices in $\Omega_{D}$. It is clear that when all edges in cutsets $s_{1}, S_{2}$ and $s_{3}$ are deleted, four connected subgraphs $g_{A}, g_{B}, g_{C}$ and $\mathrm{g}_{\mathrm{D}}$ will be produced. It is also clear from partition (1) that value $\tau_{i j}$ from any vertex $i$ in $g_{A}$ and $g_{B}$ to any vertex $j$ in $g_{C}$ and $g_{D}$ is 0 . Let $N_{A}, N_{B}$, $\mathbb{N}_{C}$ and $N_{D}$ be corresponding subnetwork of $g_{A}, g_{B}, g_{C}$ and $g_{D}$ respectively. Then $\tau_{i j}=0$ means that signal from $\mathrm{N}_{A}$ or $\mathrm{N}_{B}$ will not reach to any part of $\mathrm{N}_{C}$ and $\mathrm{N}_{D}$. Thus we can place blocks $N_{C}$ and $N_{D}$ before $N_{A}$ and $N_{B}$ to make a sequence of unidirectional blocks. By principal partition (2), we can see that $\tau_{p q}$ from any vertex $p$ in $g_{C}$ to any vertex $q$ in $g_{D}$ is 0 . Similarly, by partition (3), $\tau_{r s}$ from any vertex $r$ in $g_{A}$ to any vertex $s$ in $g_{B}$ is 0 . Thus a sequence of unidirectional blocks is $N_{D} N_{C} N_{B} N_{A}$.

The difference between unidirectional blocks and almost unidirectional blocks is that instead of $\tau_{i j}=0$ from a succeeding block to a preceding block, we have $\tau_{i j}=\varepsilon$ where $\varepsilon$ is very small. Thus Theorems 5 and 6 can be rewritten for the existance of almost unidirectional blocks by changing 0 to $E$. How small $E$ should be depends on a method of diagnosis.

\section{Conclusion:}

Here, we introduce a method of forming unidirectional blocks and almost unidirectional blocks of a network from a corresponding graph consisting of edges which indicate values of transmission. Since value of transmission from node $i$ to node $j$ is not depend only on a component connected between them but depend on almost all components in a network, a corresponding graph does not resemble structually to a given network which would be a disadvantage of this mathod. However, a corresponding graph can be obtained easily by data from suitable measurements. Hence this method may be suitable enough for helping diagnosis of large scale analog networks.

\section{References}

1. T.N. Trick, W. Mayeda \& A.A. Sakla, "Calculation of Parameter Values from Node Voltage Measurements", IEEE Trans on Circuits and Sys., vol, CAS-26, No. 7, pp 446-473, July 1979.

2. T.N. Trick, W. Mayeda \& A.A. Sakla, "Determination of Component Values From Node Vo1tage Measurements", Proc. of 1979 ISCAS, pp 878-881, July 1979.

3. S. Shinoda, Y. Kajitani, K. Onaga and W. Mayeda, "Various Characterizations of Series-Parallel Graph", Proc. of 1979 ISCAS, pp 100-103, July 1979.

4. W. Mayeda, Graph Theory, John Wiley \& Sons, 1972.

5. K. Onaga \&W. Mayeda, "A Boolean Theory of Network Flows and Matrices to particle Transmission and Clustering', Networks, vol 9 pp 249-281, 1979. 